\title{
Numerical Simulation on Circulation Flow and Mass Transfer inside Atmospheric Water Drops
}

Zhentao Wang ${ }^{\mathrm{a}, \mathrm{b}}$, Tianyu Guo ${ }^{\mathrm{a}}$, Lin Tian ${ }^{\mathrm{b}}$, Qian Xü $\mathrm{X}^{\mathrm{a}}$, Shuiqing Zhan ${ }^{\mathrm{a}}$, Jiyuan Tu ${ }^{\mathrm{b} *}$

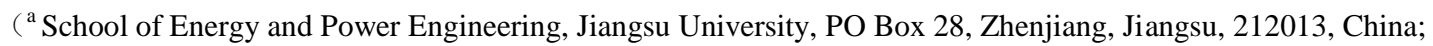

${ }^{\mathrm{b}}$ School of Aerospace, Mechanical \& Manufacturing Engineering, RMIT University, PO Box 71, Bundoora, VIC 3083, Australia )

\begin{abstract}
When a water droplet moves in atmosphere with pollutant, internal circulation is formed due to surface shear stress. This enhances internal mass transfer greatly, and improves the spray droplet $\mathrm{SO}_{2}$ absorption. In this paper, the internal circulation and diffusion of $\mathrm{SO}_{2}$ in a water droplet were numerically studied. The distribution of tangential velocity at the interface and the effect of interior circulation on sulfur dioxide transfer are analyzed under different Reynolds numbers. The numerical results indicate that there are two symmetrical vortexes inside the droplet when there is a relative motion between gas and liquid phase. The distance between the vortex core and the droplet center is around $2 / 3 R_{d}$, and the vortex velocity increases with the Reynolds numbers. The study shows sulfur dioxide absorption by the droplet is controlled by two mechanisms, w are (1) the radial diffusion due to concentration gradient; and (2) mass transport induced by internal circulation. The characteristic times of radial diffusion and vortex formation are compared. The comparison indicates that the internal circulation dominates sulfur dioxide mass transfer inside the water droplet. The internal circulation influences the sulfur dioxide mass transfer greatly with the increase of Reynolds number. On the other hand, the effect of deformation rate on mass transfer is insignificant because of the characteristic time are of the same order with the same Reynolds number.
\end{abstract}

Key words: droplet, internal circulation, VOF, sulfur dioxide, mass transfer, numerical simulation

\section{Introduction}

Due to extensive burning of fossil fuels, the atmosphere is filled with aerosol particles and air pollutants. These aerosol particles and air pollutants have caused severe environmental problems such as acid rain and haze, which have aroused extensive attention in China [1-5]. In all air pollutants, sulfur dioxide and nitrogen oxides are the major harmful elements. It is well known that acid rain is formed when sulfur dioxide and nitrogen oxides are absorbed by rain droplets. It is a great challenge to control air pollutants. Accordingly, the precise prediction on sulfur dioxide or nitrogen oxides absorption plays a vital role in understanding and controlling acid rain pollutions.

Sulfur dioxide or nitrogen oxides absorption by droplets is a series of processes related to mass transfer between gas and liquid phases. In the past several decades, a variety of theoretical models such as the film theory, penetration theory and eddy diffusion theory have been developed to describe the process of mass transfer [7-9]. The aforementioned mass transfer theories focused on the droplet interface at macro scale. The unsteady aqueous-phase oxidation of sulfur dioxide around a stagnant droplet contains five stages, which are gas-phase diffusion; mass transfer at the interface; aqueous dissociation reaction; aqueous-phase diffusion; and aqueous chemical reactions, as shown in Figure 1 [10]. Although this problem has been extensively studied adopting theoretical, experimental and numerical method and many important results have been accomplished, there are relative few studies connected to mass transfer of drops with internal circulation.

According to the published literature, internal circulation or vortex inside a drop was introduced by velocity boundary layer when the air flows around a droplet. Four approaches were used to study the internal circulation of a falling droplet. Of such, the boundary layer theory, numerical method, and experimental measurement obtained agreeable results for droplets with radii $<500 \mu \mathrm{m}$ [11]. The internal circulation in an evaporating drop was also reported in details [12-16]. There exists only a few papers concerned mass transfer of droplet with internal circulation. A convective-diffusion equation was developed to describe the gas absorption accounting the internal flow given by Hadamard-Rybczinski analytical and approximating solutions in decade years ago $[17,18]$. In recent years, the unsteady mass transfer of drops with internal circulation has been conducted as a subject in some work. Chen numerically 
investigated the unsteady absorption of sulfur dioxide, nitrogen oxides and carbon dioxide by liquid droplet accounting for the internal circulation at different airflow rate [19-21]. Extending the work, a simplified model was developed to predict the sulfur dioxide absorption by single freely falling rain drops with good accuracy [22]. Elperin theoretically investigated the air pollutants absorption processes using a system of transient partial parabolic differential equations including the combined convective diffusion in the liquid and gaseous phases with time-dependent boundary conditions $[23,24]$. The studies on air pollutants absorption by drops with internal circulation indicated that the internal circulation or interior flow could be induced by external flow around the drop and improve the heat and mass transfer. Although some prior works have gained excellent results on gas absorption, the detailed mass transfer improving mechanisms of drops with internal circulation are still not fully understood. Two types of mass-transfer mechanisms exist within the droplet: diffusion induced by the solute gradient and convective transport caused by the internal flow.

In present paper, the sulfur dioxide absorption of a full scale atmospheric droplet accounting for the droplet internal circulation at different Reynolds numbers was examined. By using VOF (Volume of Fluid) method with coupled momentum and species transport equation, the time scales associated with the exterior sulfur dioxide gas diffusion and the internal vortex formation are analyzed. In addition, the effect of the droplet deformation toward the mass transfer process, which has never been revealed, is investigated.

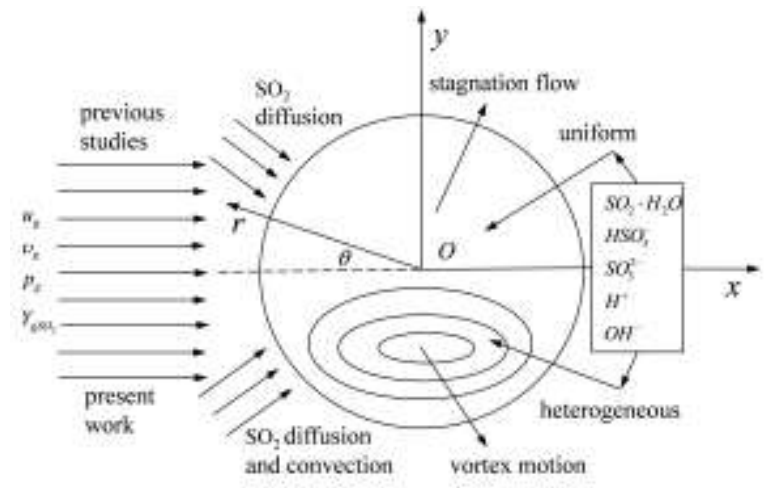

Figure 1 Schematic of the axisymmetric model of a droplet

\section{Physical Models and Mathematical Formulations}

\subsection{Problem description}

Figure 1 illustrates the 2D Schematic axisymmetric droplet model in this study. An axisymmetric liquid droplet of radius $R_{\mathrm{d}}$ travels in the atmosphere containing uniform sulfur dioxide. As the velocity boundary layer is developed, shear stress appears at the liquid-gas interface, and vortexes will form inside the droplet. The sulfur dioxide absorption by water droplet is expressed as:

$$
\mathrm{SO}_{2}+\mathrm{H}_{2} \mathrm{O}=\mathrm{SO}_{2} \cdot \mathrm{H}_{2} \mathrm{O}
$$

The current numerical simulation assumes the droplet is either spherical or elliptical under deformation. The effect of the deformation toward sulfur dioxide absorption is studied. According to published articles, the droplet keeps the original shape in this study because the Weber number is greatly below 1.1 in this paper [25]. For the flow with Reynolds number below 13.7, a laminar flow condition is assumed. Low atmospheric sulfur dioxide concentration could be considered constant. The gas species conservation equation is not needed [26]. The physical properties of the gaseous and liquid phases are constant. Fick's Law and the phase equilibrium law at the interface govern the sulfur dioxide diffusion process.

\subsection{Governing equations}

For the homogeneous and incompressible flow, the governing equations of mass, momentum, and species transfer are:

Continuity equation: 


$$
\frac{\partial \rho}{\partial t}+\frac{\partial}{\partial}(\rho u)+\frac{1}{r} \frac{\partial}{\partial r}(r \rho v)=0
$$

where $u$ is axial velocity, $v$ is radial velocity.

Axial momentum equation:

$$
\begin{aligned}
& \frac{\partial}{\partial t}(\rho u)+\frac{\partial}{\partial x}\left(\rho u u-\mu \frac{\partial u}{\partial x}\right)+\frac{1}{r} \frac{\partial}{\partial r}\left(r \rho u v-r \mu \frac{\partial u}{\partial r}\right)= \\
& -\frac{\partial p}{\partial x}+\frac{\partial}{\partial x}\left\{-\frac{2}{3} \mu\left[\frac{\partial u}{\partial x}+\frac{1}{r} \frac{\partial}{\partial r}(r v)\right]+\mu \frac{\partial u}{\partial x}\right\}+\frac{1}{r} \frac{\partial}{\partial r}\left(r \mu \frac{\partial v}{\partial x}\right)
\end{aligned}
$$

Radial momentum equation:

$$
\begin{aligned}
& \frac{\partial}{\partial t}(\rho v)+\frac{\partial}{\partial x}\left(\rho u v-\mu \frac{\partial v}{\partial x}\right)+\frac{1}{r} \frac{\partial}{\partial r}\left(r \rho v v-r \mu \frac{\partial v}{\partial r}\right)= \\
& -\frac{\partial p}{\partial r}+\frac{1}{r} \frac{\partial}{\partial x}\left\{r \mu \frac{\partial v}{\partial x}-\frac{2}{3} r \mu\left[\frac{\partial u}{\partial x}+\frac{1}{r} \frac{\partial}{\partial r}(r v)\right]\right\}+\frac{\partial}{\partial x}\left(\mu \frac{\partial u}{\partial r}\right)-2 \mu \frac{v}{r^{2}}+\frac{2}{3} \mu \frac{1}{r}\left(\frac{\partial u}{\partial x}+\frac{1}{r} \frac{\partial}{\partial r}(r v)\right)
\end{aligned}
$$

Species equation in liquid phase:

$$
\frac{\partial}{\partial}\left(\rho_{l} Y_{l}\right)+\frac{\partial}{\partial x}\left(\rho_{l} u_{l} Y_{l}-\rho_{l} D_{l} \frac{\partial Y_{l}}{\partial x}\right)+\frac{1}{r} \frac{\partial}{\partial r}\left(r \rho_{l} v_{l} Y_{l}-r \rho_{l} D_{l} \frac{\partial Y_{l}}{\partial r}\right)=0
$$

where $Y_{l}$ is the liquid molar fraction, $D_{l}$ is the liquid diffusivity.

1.3 Initial and boundary conditions

The initial conditions for gas and liquid phase are:

$$
\begin{gathered}
u_{g}=u_{g \infty}, \quad v_{g}=0, \quad p_{g}=p_{g \infty}, \text { and } Y_{g, S O_{2}}=Y_{g, S O_{2} \infty} \\
u_{l}=0, \quad v_{l}=0, \quad p_{l}=p_{g \infty}, \text { and } Y_{l, S O_{2}}=0
\end{gathered}
$$

In the spherical coordinate $\left(r_{\mathrm{n}}, \theta\right)$, the boundary conditions are:

(i) Upstream inflow $\left(r_{\mathrm{n}}=r_{\infty}, 0 \leq \theta \leq \pi / 2\right)$ :

$$
u_{g}=u_{g \infty}, v_{g}=0, p_{g}=p_{g \infty}, Y_{g, S O_{2}}=Y_{g, S O_{2 \infty}}
$$

(ii) Downstream outflow $\left(r_{\mathrm{n}}=r_{\infty}, \pi / 2 \leq \theta \leq \pi\right)$ :

$$
\frac{\partial u_{g}}{\partial r_{n}}=\frac{\partial v_{g}}{\partial r_{n}}=\frac{\partial p_{g}}{\partial r_{n}}=0, Y_{g, S O_{2}}=Y_{g, S O_{2} \infty}
$$

(iii) Axis of symmetry $\left(0 \leq r_{\mathrm{n}} \leq r_{\infty}, \theta=0\right.$ or $\left.\theta=\pi\right)$ :

$$
v_{g}=\frac{\partial u_{g}}{\partial \theta}=\frac{\partial p_{g}}{\partial \theta}=0, Y_{g, S O_{2}}=Y_{g, S O_{2} \infty}, v_{l}=\frac{\partial u_{l}}{\partial \theta}=\frac{\partial p_{l}}{\partial \theta}=\frac{\partial Y_{l, S O_{2}}}{\partial \theta}=0
$$

(iv) Droplet surface $\left(r_{\mathrm{n}}=r_{\mathrm{s}}\right)$

$$
v_{g \theta}=v_{l \theta}, \mu_{g}\left(\frac{\partial v_{g \theta}}{\partial r_{n}}-\frac{v_{g \theta}}{r_{s}}+\frac{1}{r_{s}} \frac{v_{g}}{\partial \theta}\right)=\mu_{l}\left(\frac{\partial v_{l \theta}}{\partial r_{n}}-\frac{v_{l \theta}}{r_{s}}+\frac{1}{r_{s}} \frac{v_{1}}{\partial \theta}\right), p_{g}=p_{l}
$$

The phase equilibrium is assumed at the interface, and Henry's law, governing the gas absorption rate under atmospheric pressure, is expressed as below:

$$
\left[\mathrm{SO}_{2} \cdot \mathrm{H}_{2} \mathrm{O}\right]=\mathrm{H}_{\mathrm{SO}_{2}} \cdot p_{\mathrm{SO}_{2}}
$$

In Eq. (12), $p_{\mathrm{SO} 2}$ represents the partial pressure of $\mathrm{SO}_{2}$ in the atmosphere, and $H_{\mathrm{SO} 2}$ is the coefficient of physical solubility of $1.26 \mathrm{M} / \mathrm{atm}$. 


\section{Numerical Methods}

In this study, by placing the coordinate on the droplet, the process could be viewed as the air containing sulfur dioxide passing through a stationary droplet. The gas and liquid phase equations are solved by VOF (Volume of Fluid) method. In the VOF model, all fluids share the same momentum equation. Volume fraction of each phase in each cell is then calculated. The fluid properties in every cell are calculated by the volume-weighted method.

$$
\begin{aligned}
& \rho=\alpha_{1} \rho_{1}+\left(1-\alpha_{1}\right) \rho_{2} \\
& \mu=\alpha_{1} \mu_{1}+\left(1-\alpha_{1}\right) \mu_{2}
\end{aligned}
$$

where, $\alpha_{1}$ and 1- $\alpha_{1}$ are the fluid volume fractions, $\rho$ and $\mu$ are the density and dynamic viscosity of the fluids respectively.

In this study, the solution is obtained by solving the momentum and species transport equations using the commercial software Fluent. The mesh is generated with Gambit. The governing equations are discretized into the algebraic counterparts based on the finite volume method [27]. A semi-implicit method for pressure-linked equations was adopted to solve the flow field. First order upwind scheme was employed for convective and diffusive fluxes over the control volume surface. An orthogonal grid system was constructed to perform the simulation (Figure 2). The orthogonal grid system uses fewer grids, reduces truncation errors, and is beneficial at the droplet surface. The iteration time step is chosen to be $10^{-5} \mathrm{~s}$.

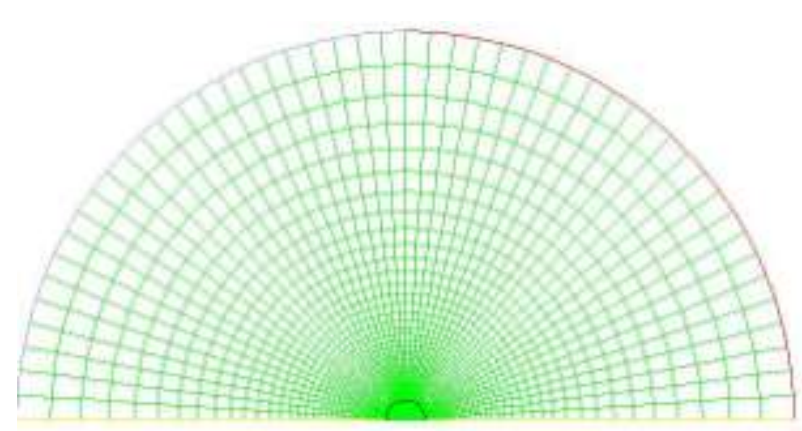

(a)

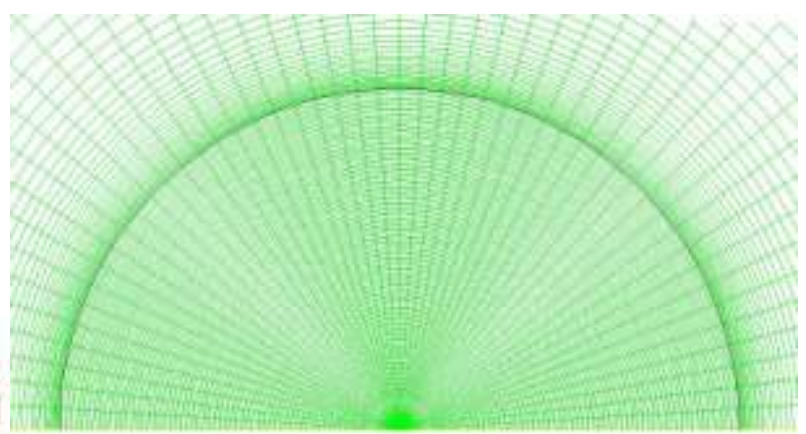

(b)

Figure 2 (a) The orthogonal system adopted in this study, and (b) the detailed grids distribution near the droplet surface

The accuracy of the numerical calculation is greatly affected by the mesh quality. A grid independence study was performed. The three grid systems of 8300, 23200 and 53823 elements are applied at $R_{\text {eg }}=40$ (Figure 2(a) and (b)) Figure 3(a) shows the comparison of the predicted pressure coefficient ( $C_{\mathrm{p}}$, Eq. (15)) along a cylinder surface for the case of $R_{\mathrm{eg}}=40$. It is clearly shown that the three grids obtained the similar results. In order to save the memory and computing time, the number of grids is set to be 8300. A comparison of the pressure distribution along a cylinder surface at $R_{\mathrm{eg}}=40$ with prior studies are also performed and a good agreement was obtained [28]. The present model is shown to be accurate to predict convective behaviors of a droplet.

$$
C_{p}=\frac{2\left(p_{s}-p_{\infty}\right)}{\rho_{g} u_{\infty}^{2}}
$$




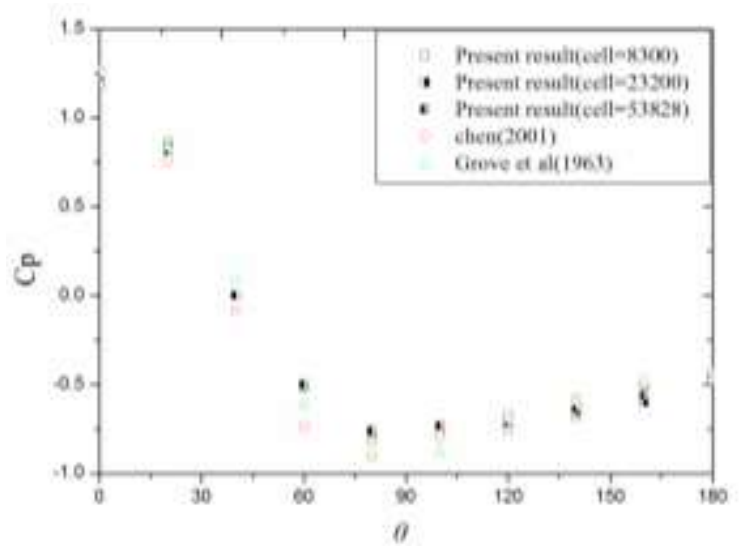

Figure 3 Grid independence and model validation

\section{Results and Discussion}

It is shown from the study that the distance between the droplet center and the center of the internal vortex is around $2 / 3 R_{\mathrm{d}}$. The physical parameters of the fluids and the diffusion coefficients are listed in Table 1 . Meanwhile, the operating parameters of the simulation are listed in Table 2. In the current study, three different Reynolds numbers of $R_{\text {eg }}=0.137,1.37$, and 13.7, corresponding to the droplet with velocities of $u_{\infty}=0.1,1.0$, and $10.0 \mathrm{~m} / \mathrm{s}$, are employed. The temperature is $293 \mathrm{~K}$, and the pressure is $1.01325 \times 10^{5} p_{\mathrm{a}}$.

Table 1. Physical parameters of fluids and the diffusion coefficients

\begin{tabular}{cccccc}
\hline$\rho_{g}\left(\mathrm{~kg} / \mathrm{m}^{3}\right)$ & $\rho_{l}\left(\mathrm{~kg} / \mathrm{m}^{3}\right)$ & $\mu_{\mathrm{g}}(\mathrm{kg} / \mathrm{m} \cdot \mathrm{s})$ & $\mu_{l}\left(p_{\mathrm{a}} \cdot \mathrm{s}\right)$ & $D_{g}\left(\mathrm{~m}^{2} / \mathrm{s}\right)$ & $D_{l}\left(\mathrm{~m}^{2} / \mathrm{s}\right)$ \\
\hline 1.225 & 998.2 & $1.7894 \times 10^{-5}$ & $1.003 \times 10^{-3}$ & $1.08 \times 10^{-5}$ & $1.47 \times 10^{-9}$ \\
\hline
\end{tabular}

Table 2 Specific operating parameters

\begin{tabular}{ccc}
\hline$R_{\mathrm{d}}(\mu \mathrm{m})$ & $u_{\infty}(\mathrm{m} / \mathrm{s})$ & $R_{e \mathrm{~g}}$ \\
& 0.1 & 0.137 \\
\multirow{2}{*}{10.0} & 1.0 & 1.37 \\
& 10.0 & 13.7 \\
\hline
\end{tabular}

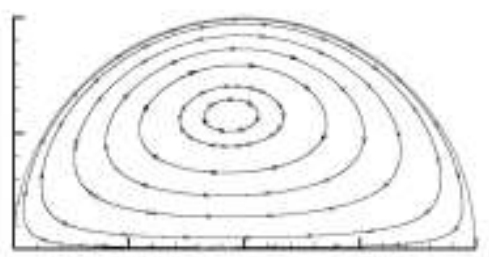

(a)

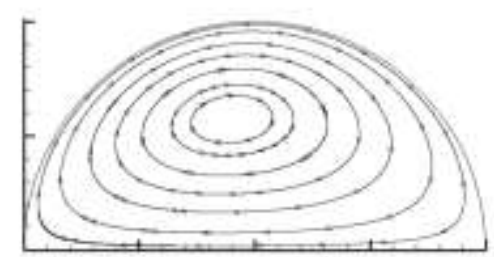

(b)

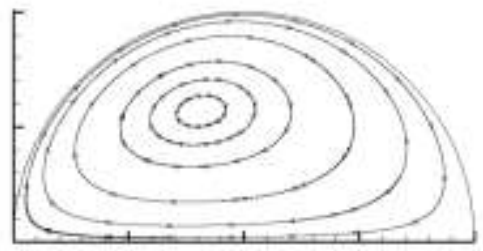

(c)

Figure 4 Circulation inside the droplet

(a) $\mathrm{R}_{\text {eg }}=0.137$; (b) $\mathrm{R}_{\mathrm{eg}}=1.37$; (c) $\mathrm{R}_{\text {eg }}=13.7$ 


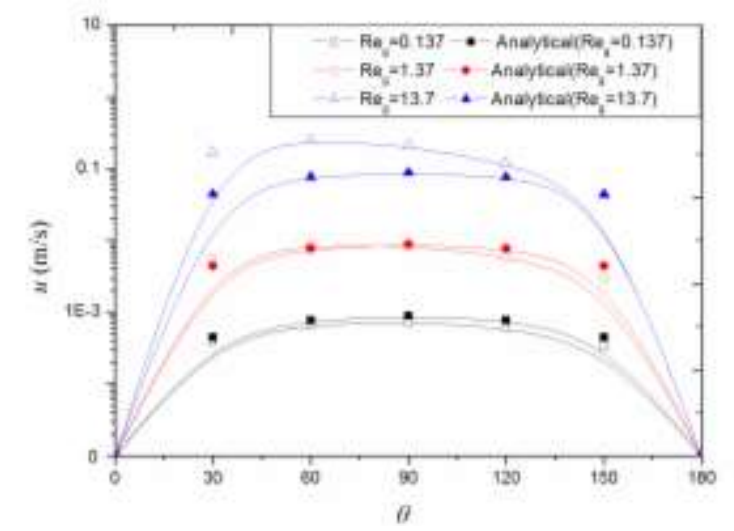

Figure 5 Distributions of droplet surface tangential velocity

\subsection{Droplets internal velocity}

Due to the high shear stress at the liquid-gaseous interface, internal circulation is formed in the droplet. Simulations of the internal vortex under different Reynolds numbers are showed in Figure 4. A distance of about $2 / 3 R_{\mathrm{d}}$ between the center of the vortex and the droplet is observed. This is consistent with the analytical solution and prior studies. With the increase of the gas velocity, larger velocity gradient in the boundary layer will form, and larger shear stress will be induced. As a result, vortex velocity will increase accordingly. Figure 5 shows the predicted and analytical surface velocity at different Reynolds number. An approximated sinusoidal function of the surface velocity is observed in Figure 5 and the strength of the internal vortex could be characterized by the droplet interfacial velocity. The predicted values are very close to analytical values under very low Reynolds number, while have some discrepancy in larger Reynolds number because the analytical solution is only for low Reynolds numbers. The diameter perpendicular to the flow direction is regarded as the symmetrical axis, and this results in a front and rear sections of the droplet. As seen from the figure, the velocity distribution in the droplet is not symmetric in the front and rear sections. A larger velocity distributed is observed in the front section, while a lower one appears in the rear section. This is caused by an unsymmetrical friction experienced by the front and rear sections of the droplet, and more significant to the droplet at higher Reynolds number.

\section{2 $\mathrm{SO}_{2}$ interfacial concentration}

The time evolving $\mathrm{SO}_{2}$ concentration at the interfacial point of $r=R_{\mathrm{d}}, \theta=90^{\circ}$ at different Reynolds number is presented in Figure 6. It is shown that the concentration decreased significantly with the increase of Reynolds number, especially from 1.37 to 13.7. This might related to the strengthening of the internal circulation and the enhanced $\mathrm{SO}_{2}$ absorption by the internal convection. $\mathrm{SO}_{2}$ at the droplet surface was rapidly carried to the center by the circulation vortex. The internal circulation appears to reduce the concentration gradient from the droplet surface to the vortex center, enhance the $\mathrm{SO}_{2}$ mass transfer, and improve the $\mathrm{SO}_{2}$ uptake by the water droplet. The concentration level is more stable at higher Reynolds number. The reason might be that it took longer time to form the internal circulation vortex at lower Reynolds number. It is shown that the concentration for Reynolds number of 0.137 and 1.37 departed with time. It is also observed that for all cases, the concentration increases with the time. In the present study, the atmospheric $\mathrm{SO}_{2}$ concentration outside the droplet is assumed constant. The $\mathrm{SO}_{2}$ will be continuously carried to the droplet center through the interfacial and internal processes. The decrease of $\mathrm{SO}_{2}$ concentration in the atmosphere due to the droplet uptake is neglected in the current study. 


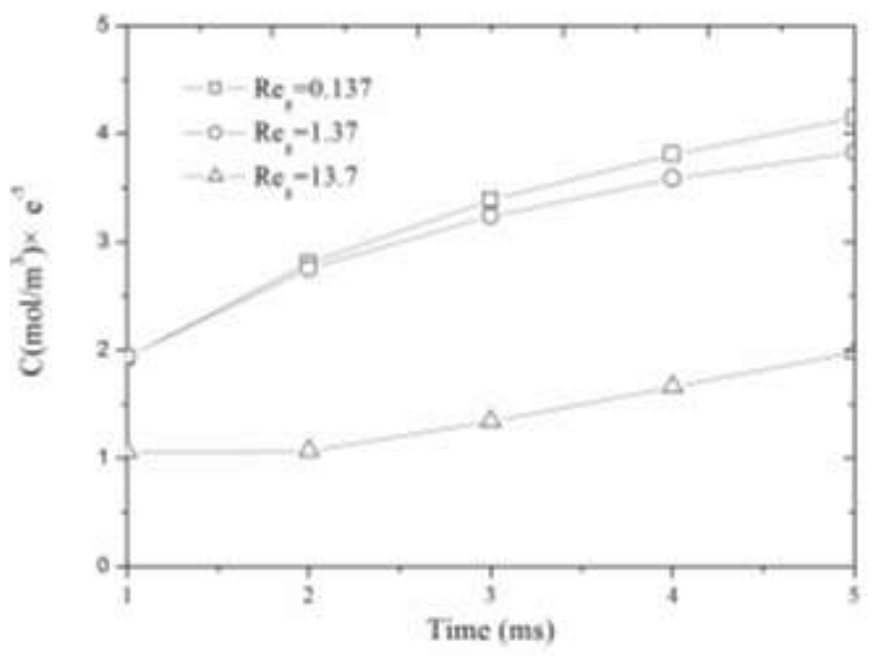

Figure $6 \mathrm{SO}_{2}$ concentrations at the interface

\subsection{Effect of internal circulation on the $\mathrm{SO}_{2}$ mass transfer}

Uptake of the $\mathrm{SO}_{2}$ by the water droplet is a non-stationary process in the present study. Two-stage mass transfer mechanisms occur in the entire process. First one is the radial diffusion across the gas-liquid interface towards the droplet center driven by the sulfur dioxide concentration gradient in the radial direction. The second mass transfer mechanism is the vortex convection inside the droplet. This vortex convection tends to decrease the sulfur dioxide concentration at the droplet surface and brings even the $\mathrm{SO}_{2}$ concentration inside the droplet. Figure 7 shows the sulfur dioxide concentration together with the flow streamlines at different Reynolds number. At low Reynolds number $(\operatorname{Re}=0.137)$, sulfur dioxide mass transfer appears to mainly occur in the radial direction, and the mass transfer rate is considerably slow. Over time, the sulfur dioxide concentration propagates toward the radial direction and the lowest concentration is always located around the droplet center, (Figure 7(a)). It is clearly shown that, at low Reynolds number, the sulfur dioxide mass transfer is dominated by the radial diffusion driven by the concentration gradient, and it takes relatively long time. When the Reynolds number is increased to 1.37 , the improved vortex motion enhances the sulfur dioxide mass transfer. It is clearly shown from Figure 7(b) that the sulfur dioxide uptake is not only in the droplet radial direction, but also coupled with the internal vortex motion. The mass transfer is clearly influenced by a two-stage transport process. The lowest concentration is not located at the droplet center. Instead, it is evolving and coupled with the internal circulation. In addition, the vortex velocity in the front section is larger than that of the rear section, and the $\mathrm{SO}_{2}$ concentration resembles the velocity profile in the rear section. The asymmetric concentration distribution in the front and rear sections enhance as time evolves. Figure 7(c) shows the sulfur dioxide concentration distributions at different times as the Reynolds number is increased to 13.7. It is clearly shown that the concentration contour core departs from the droplet center and migrates to the vortex center. The concentration contour coincident with the flow streamlines and the lowest concentration is located at the vortex center. In this case, the mass transfer of $\mathrm{SO}_{2}$ dominantly driven by the internal convection towards the vortex center. The results indicate that the enhanced vortex motion due to higher Reynolds number improves the sulfur dioxide mass transfer, and make even the sulfur dioxide concentration inside the droplet. The vortex motion therefore increases the sulfur dioxide uptake towards the vortex core. The two-stage mass transfer process is shown to enhance sulfur dioxide absorption rate. As a result, the interfacial $\mathrm{SO}_{2}$ concentration gradually decreases with the increase of the Reynolds number and correlates to the results shown in Figure 6.
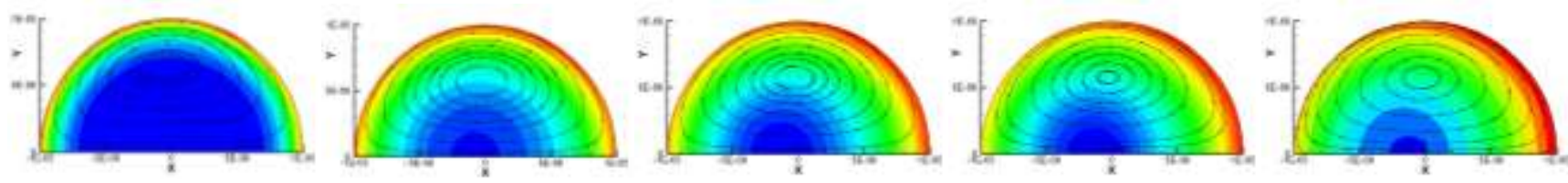
$t=1 \mathrm{~ms}$

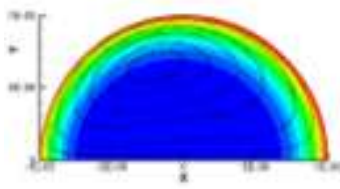

$t=1 \mathrm{~ms}$

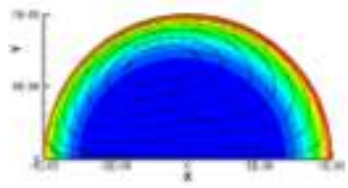

$t=1 \mathrm{~ms}$ $t=5 \mathrm{~ms}$

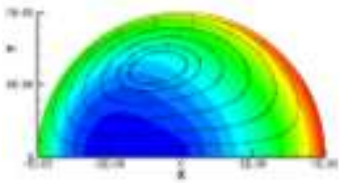

$t=5 \mathrm{~ms}$

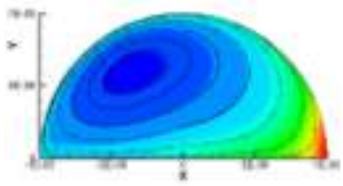

$t=5 \mathrm{~ms}$ $t=10 \mathrm{~ms}$

(a) $R \mathrm{e}_{\mathrm{g}}=0.137$ $t=15 \mathrm{~ms}$

$t=20 \mathrm{~ms}$

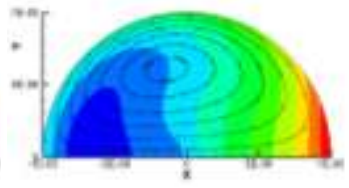

$t=10 \mathrm{~ms}$

(b) $R \mathrm{e}_{\mathrm{g}}=1.37$

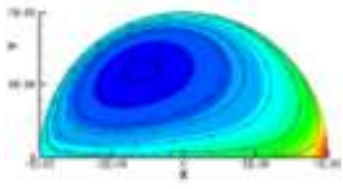

$t=10 \mathrm{~ms}$

(c) $R \mathrm{e}_{\mathrm{g}}=13.7$

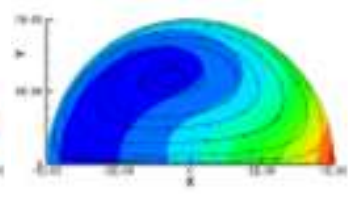

$t=15 \mathrm{~ms}$

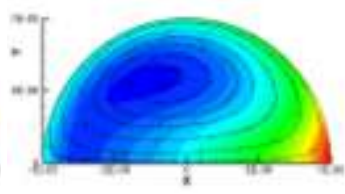

$t=20 \mathrm{~ms}$

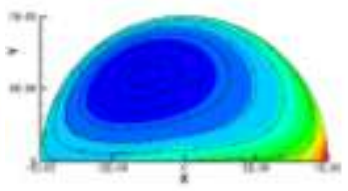

$t=15 \mathrm{~ms}$

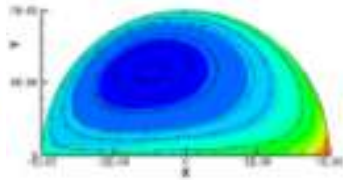

$t=20 \mathrm{~ms}$

Figure $7 \mathrm{SO}_{2}$ concentration distributions in the droplet at various Reynolds numbers and times

\subsection{Effect of droplet deformation on the $\mathrm{SO}_{2}$ mass transfer}

A moving droplet in the air could deform. The deformation of the droplet could be described by a spheroid with different aspect ratios $(e=a / b$, here $a$ and $b$ are the equatorial and polar radii of the spheroid droplet). An aspect ratio of $e$ $=1.5$ is analyzed and shown in Figure 8. The numerical results indicated that the internal circulation and mass transfer patterns of the deformed droplet resemble that of the spherical droplet at different Reynolds numbers. The $\mathrm{SO}_{2}$ uptake is determined by two factors: (1) the radial diffusion due to concentration gradient; and (2) mass transfer driven by the internal circulation. At Reynolds number of $0.137,1.37$ and 13.7, the $\mathrm{SO}_{2}$ is governed by a combination of the mass diffusion in the radial direction due to concentration gradient and the mass convection due to fluid internal circulation. While the first mechanism dominates the process at low Reynolds number $\left(R \mathrm{e}_{\mathrm{g}}=0.137\right)$, the latter dominates at a higher Reynolds number $\left(R \mathrm{e}_{\mathrm{g}}=13.7\right)$. When compared to that of the spherical droplet, the center of the internal circulation of the deformed droplet is closer to the gas-liquid interface, which might imply an enhanced diffusion mass transfer due to higher concentration gradient. However, the overall mass transfer outcome should be a combined effect of the change in mass diffusion and convection. A series of different deformation rates are considered and similar results are obtained.

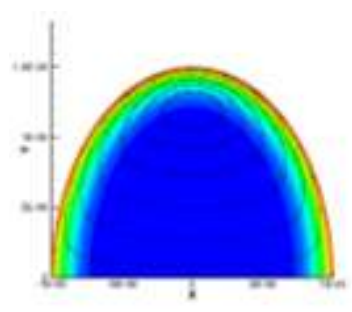

$t=1 \mathrm{~ms}$

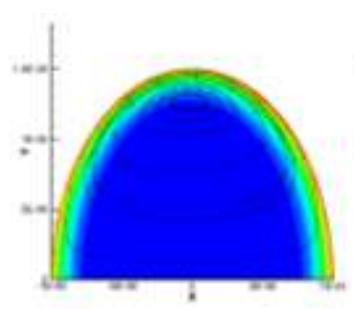

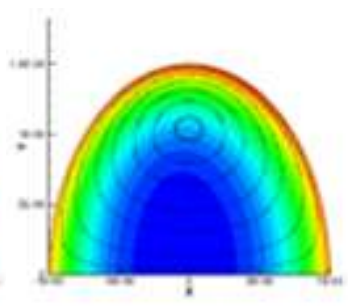

$t=5 \mathrm{~ms}$

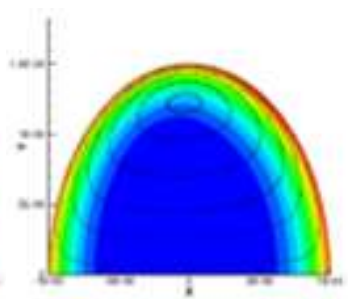

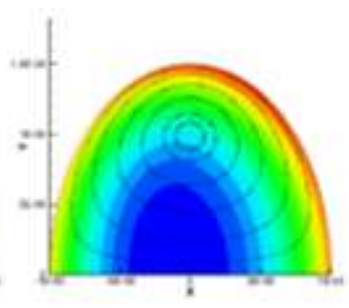

$t=10 \mathrm{~ms}$

(a) $R \mathrm{e}_{\mathrm{g}}=0.137$

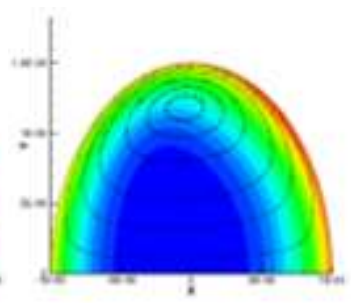

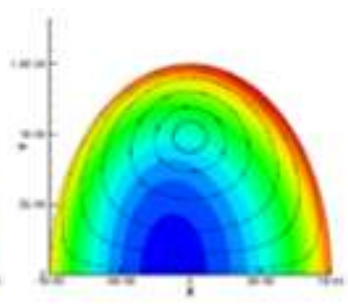

$t=15 \mathrm{~ms}$

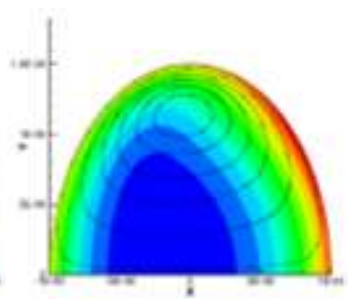

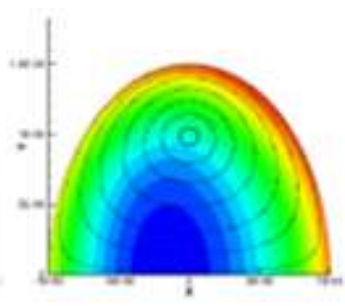

$t=20 \mathrm{~ms}$

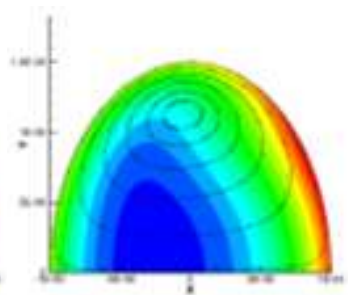


$t=1 \mathrm{~ms}$

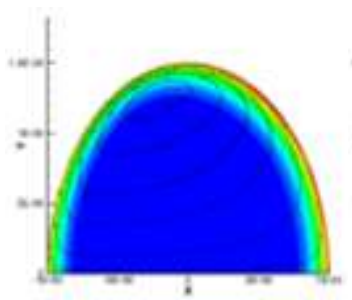

$t=1 \mathrm{~ms}$ $t=5 \mathrm{~ms}$

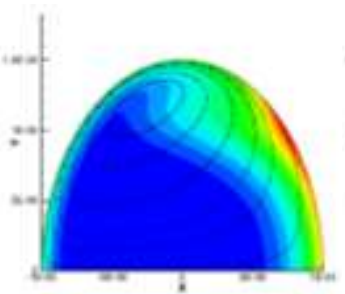

$t=5 \mathrm{~ms}$ $t=10 \mathrm{~ms}$

$t=15 \mathrm{~ms}$

(b) $R \mathrm{e}_{\mathrm{g}}=1.37$

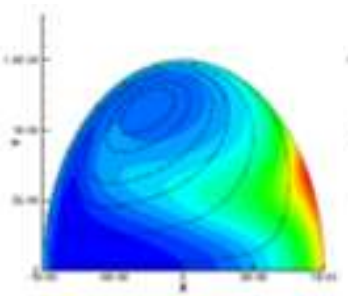

$t=10 \mathrm{~ms}$

c) $R \mathrm{e}_{\mathrm{g}}=13.7$

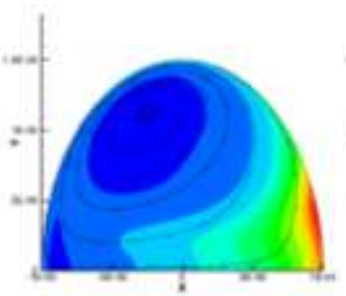

$t=15 \mathrm{~ms}$ $t=20 \mathrm{~ms}$

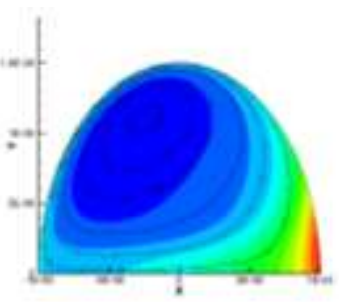

$t=20 \mathrm{~ms}$

Figure $8 \mathrm{SO}_{2}$ concentration distributions in the droplet at various Reynolds numbers and times for spheroid droplet.

\subsection{Time scale analysis}

\subsubsection{Spherical droplet}

The effect of the vortex motion on $\mathrm{SO}_{2}$ mass transfer rate could be evaluated by the comparison of the time scales associated with the vortex formation and the radial diffusion. The characteristic time for a steady state vortex formation is given by Parakash and Sirignano as[29]:

$$
t_{\text {vortexformation }}=o\left(\frac{R_{d}{ }^{2}}{v_{l}}\right)
$$

where $v_{l}$ is the kinematic viscosity of water and $\mathrm{R}_{\mathrm{d}}$ is the water droplet radius.

If the droplet is assumed to be stationary, namely, lump system, the radial diffusion characteristic time could be estimated as [19]:

$$
t_{\text {mass diffusion }}=o\left(\frac{R_{d}{ }^{2}}{D_{l}}\right)
$$

The Schmidt number, a ratio between the characteristic times of the mass diffusion and the vortex formation, for the spherical droplet is:

$$
\frac{t_{\text {mass diffusion }}}{t_{\text {vortex formation }}}=o\left(\frac{R_{d}{ }^{2} / v_{l}}{R_{d}{ }^{2} / D_{l}}\right)=o\left(\frac{v_{l}}{D_{l}}\right)=o\left(S c_{l}\right)=o(683.54)
$$

According to the Schmidt number, it is clearly shown that the characteristic time of vortex formation is much shorter than that of the radial diffusion. With a strong vortex motion, the lowest $\mathrm{SO}_{2}$ concentration is at the vortex center coincident with the mass transfer center. This implies a mass diffusion distance of one third the droplet radius from the liquid-gas surface to the mass transfer center driven by the concentration gradient. In this situation, the effect of vortex motion on $\mathrm{SO}_{2}$ uptake must be included. As a result, the modified characteristic time of the radial diffusion with vortex motion, and the Schmidt number, are,

$$
\begin{gathered}
t^{*}{ }_{\text {massdiffusion }}=o\left(\frac{\left(R_{d}^{*}\right)^{2}}{D_{l}}\right) \\
\frac{t_{\text {mass diffusion }}^{*}}{t_{\text {vortex formation }}}=o\left(\frac{v_{l}}{9 D_{l}}\right)=o(75.95)
\end{gathered}
$$

In Eq. (19), $R_{\mathrm{d}}{ }^{*}$ is the characteristic length and equals to $1 / 3 R_{\mathrm{d}}$. With the inclusion of the vortex motion, time scale of 
the $\mathrm{SO}_{2}$ mass diffusion decreases significantly. However, the characteristic time of the vortex motion formation is still significantly shorter than that of the radial diffusion. Eq. (18) and (20) imply an unsteady developing stage with the onset of the vortex formation till a fully developed internal vortex. The vortex motion always influences the $\mathrm{SO}_{2}$ mass transfer in the entire period.

According to the previous study and above analysis, the $\mathrm{SO}_{2}$ mass transfer is dominated by two mass transfer mechanisms: (1) radial diffusion; and (2) internal circulation. The mass transfer due to internal circulation will be enhanced with the increase of the Reynolds number, and dominates the entire $\mathrm{SO}_{2}$ uptake. The characteristic time of the droplet internal circulation could be expressed as [30]:

$$
t_{\text {internalcirculatia }}=o\left(\frac{2 R_{d}}{u_{s}}\right)
$$

where $u_{s}$ is the characteristic internal velocity. In the current study, $u_{s}$ is assumed to be the maximum velocity on the droplet surface. The ratio of the characteristic time of the mass diffusion and the internal circulation could be described as:

$$
\alpha^{*}=\frac{t_{\text {mass diffusion }}}{t_{\text {int ernal circulation }}}=o\left(\frac{\left(R_{d}^{*}\right)^{2} / D_{l}}{2 R_{d} / u_{s}}\right)=o\left(\frac{\left(R_{d}^{*}\right)^{2} u_{s}}{2 R_{d} D_{l}}\right)
$$

In the present study, when the Reynolds number is 0.137 , a characteristic velocity $u_{\mathrm{s}}$ is approximately $7.6 \times 10^{-4} \mathrm{~m} / \mathrm{s}$ and the characteristic ratio is in the order of 0.287 . The result indicates that the characteristic time of the mass diffusion is shorter than $\mathrm{SO}_{2}$ uptake by internal circulation. Therefore, the mass transfer is dominated by the mass diffusion, and the concentration contour shows concentric circles. This correlates well with the prediction of Figure 7(a). In contrast, when the Reynolds number is 13.7 , a typical velocity $u_{\mathrm{s}}$ is approximately $2.6 \times 10^{-1} \mathrm{~m} / \mathrm{s}$ and the characteristic time ratio is in the order of 98.262. The characteristic time ratio in this case indicates that the characteristic time of mass diffusion is much longer than the $\mathrm{SO}_{2}$ uptake with internal circulation. Therefore, the $\mathrm{SO}_{2}$ mass transfer is dominated by the internal circulation, as shown in Figure 7(c). The lowest concentration is located at the vortex center, and the concentration contour is coincident with the internal flow streamlines. For the intermediate Reynolds number of 1.37, a typical velocity is $8.8 \times 10^{-3} \mathrm{~m} / \mathrm{s}$, and the characteristic time ratio is 3.326 . The two characteristic time scales have the same order of magnitude. This indicates that the mass transfer of $\mathrm{SO} 2$ by a water droplet is controlled by both the radial mass diffusion and the internal circulation. In this case, the concentration contour shows both a radial diffusion and an internal convection as shown in Figure 7(b).

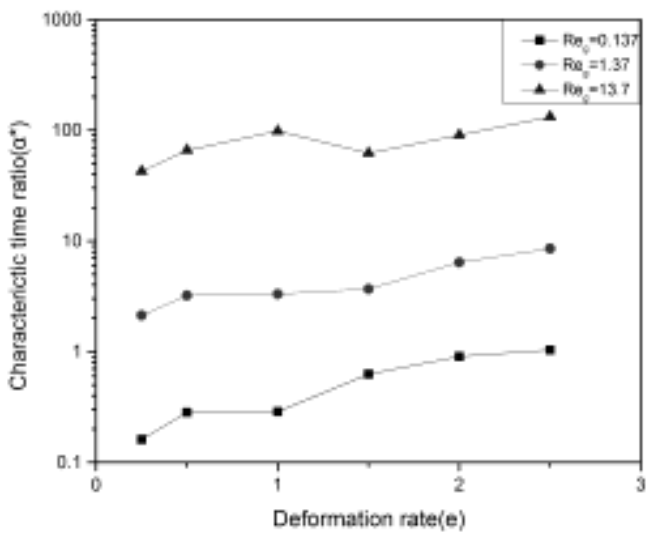

(a)

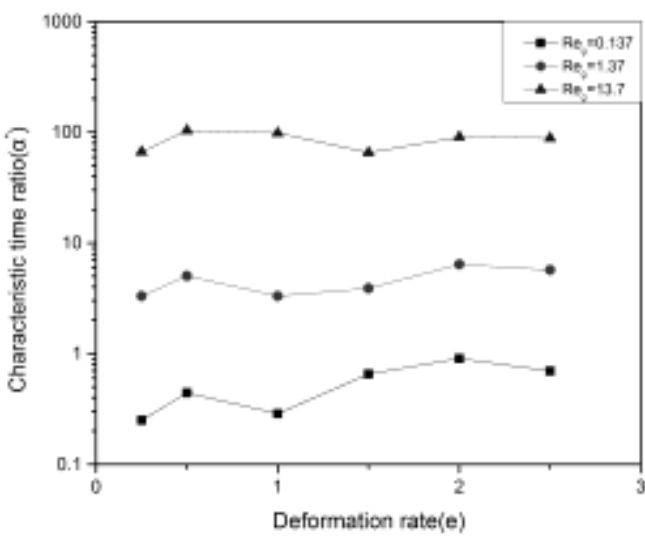

(b)

Figure 9 The ratio of characteristic time for different deformation rates 
Table 3 The distance between the vortex core and at different deformation rates

\begin{tabular}{ccccccc}
\hline$e$ & 0.25 & 0.5 & 1 & 1.5 & 2 & 2.5 \\
$R_{\mathrm{d}}^{*} / R_{\mathrm{d}}$ & 0.417 & 0.417 & 0.333 & 0.342 & 0.333 & 0.273 \\
\hline
\end{tabular}

\subsubsection{Deformed droplet}

For a deformed droplet, $R_{\mathrm{d}}$ is set to be the polar radius. $R_{\mathrm{d}}{ }^{*}$ is the characteristic length of the concentration center and assumed to be equal to $1 / 3 R_{\mathrm{d}}$. The characteristic mass transfer time ratio for the deformed droplets at different deformation rates is obtained using Eq. (25), and shown in Figure 9(a). It is found that at the same deformation rate, the characteristic time ratio increases with the increase of Reynolds number. This resembles that of the spherical droplet that the increased Reynolds number indicate enhanced droplet internal circulation, and therefore, mass transfer by convection is increased. Similar to the spherical droplet, for a Reynolds number of 0.137 , the diffusion dominates the mass transfer, while at Reynolds number of 13.7, the internal circulation governs the mass transfer process. At lower Reynolds numbers ( 0.137 or 1.37), the characteristic time ratio increases with the increase of the deformation rate. This might be attributed to the reduced internal circulation formation time, and the strengthening of the internal circulation on the mass transfer process. At the same Reynolds number, the characteristic droplet surface velocity increases with the increase of deformation rate, therefore, the shear stress at the droplet surface increases. This could decrease the droplet internal circulation formation time, and result in an increased characteristic time ratio. For larger Reynolds number of $\mathrm{Re}=13.7$, a setback of the characteristic time ratio is observed from aspect ratio of 1.0 to 1.5 , indicating a change of the deformation from oblate to prolate, which might be related to the frictional consequence significant to the high Reynolds number droplet. In addition, it is found out that high deformation rate implies a reduced distance between the vortex core and the droplet surface that, it is no longer $1 / 3 R_{\mathrm{d}}$. The distance between the center of the vortex and the droplet is showed in Tables 3 , and it is seen that an approximation of $1 / 3$ is acceptable when the deformation rate is in between 1.0 or 2.0. The characteristic time ratio $\alpha^{*}$ of different deformation rate is calculated by using $R_{\mathrm{d}}{ }^{*}$ listed in table 3 instead of $1 / 3 R_{\mathrm{d}}$, and is shown in Figure 9 (b). When compared to Figure 9(a), the variation of the characteristic time ratio with respect to the deformation rate shows similar trend however less obvious. Therefore, it is safe to state that the deformation of water droplet in the considered range has little effect on the mass transfer characteristic time ratio, or the influence of the droplet deformation on mass transfer is negligible.

\section{Conclusions}

Two symmetrical vortexes will be formed inside the liquid droplet due to the flow shear between the gas and liquid phases. The center of vortex motion is located at $2 / 3 \mathrm{R}_{\mathrm{d}}$ from the droplet center. The mass transfer of the atmospheric $\mathrm{SO}_{2}$ is controlled by two mechanisms which are (1) radial diffusion; and (2) vortex convection. The time scale of vortex formation is much faster than diffusion therefore the mass transfer is always affected by vortex motion. When the Reynolds number is small, the radial mass diffusion dominates the mass transfer, while at high Reynolds number, internal circulation controls the $\mathrm{SO}_{2}$ uptake process of. At intermediate Reynolds number, diffusion and internal circulation have comparative effect on the $\mathrm{SO}_{2}$ mass transfer, and vortex motion clearly enhances the mass transfer. On the other hand, a deformation rate of 0.25 to 2.5 has minimum influence on $\mathrm{SO}_{2}$ uptake by a liquid droplet.

\section{Acknowledgments}

This work is supported by the National Natural Science Foundation of China (51106064), The Priority Academic Program Development of Jiangsu Higher Education Institutions (PAPD), and Jiangsu University Supporting Project for NSFC (FCJJ2015001). 


\section{References}

[1] Chen D, Liu X, Lang J, Zhou Y, Wei L, Wang X, Guo X. Estimating the contribution of regional transport to PM 2.5 air pollution in a rural area on the North China Plain. Science of the Total Environment, 2017, 583, 280-291.Wang H M,

[2] Lai S, Zhao Y, Ding A, Zhang Y, Song T, Zheng J, Ho K F, Lee S, Zhong L. Characterization of pm 2.5, and the major chemical components during a 1-year campaign in rural guangzhou, southern china. Atmospheric Research, 2016, 167, 208-215.

[3] Zhao H B, Wang K, Zheng C G. Simulating and modeling particulate removal processes by elliptical fibers [J]. Aerosol Science and Technology, 2014, 48(2), 207-218.

[4] Chen H, Zheng Z, Chen Z, Bi X T. Simulation of flow and heat transfer around a heated stationary circular cylinder by lattice gas automata [J]. Powder Technology, 2016, 290:72-82.

[5] Wang Z T. Experimental investigation on wet flue gas desulfurization with electrostatically-assited twin-fluid atomization. Environmental Engineering and Management Journal, 2013, 12(9), 1861-1868.

[6] Lewis W K, Whitman W G. Principles of gas absorption. Industrial and Engineering Chemistry, 1924, 16(12): 1215-1220.

[7] Higbie R. The rate of absorption a pure gas into a still liquid during short periods of exposure. Transactions of the American Institute of Chemical Engineers, 1935, 31: 365-377.

[8] Danckwerts P V. Significance of liquid-film coefficients in gas absorption. Engineering and Process Development, 1951, 43(6): 1460-1467.

[9] King C J. Turbulent liquid phase mass transfer at a free gas-liquid interface. Industrial and Engineering Chemistry Fundamentals, 1966, 5(1): 1-16.

[10] Schwartz S E, Freiberg J E. Mass-transport limitation to the rate of reaction of gases in liquid droplets: Application to oxidation of $\mathrm{SO}_{2}$ in aqueous solutions. Atmospheric Environment, 1981, 15(7): 1129-1144.

[11] Leclair B P, Hamielec A E, Pruppacher H R, Hall W D. Theoretical and experimental study of the internal circulation in water drops falling at terminal velocity in air. Journal of the Atmospheric Sciences, 1972, 29: 728-740.

[12] Prakash S, Sirignano W A. Liquid fuel droplet heating with internal circulation. International Journal of Heat and Mass Transfer, 1978, 21(7), 885-895.

[13] Zhang N, Yang W J. Natural-convection in evaporating minute drops. Journal of Heat Transfer-Transactions of the ASME 1982, 104(4), 656-662.

[14] Hegseth J J, Rashidnia N, Chai A. Natural convection in droplet evaporation. Physical Review E 1996, 54(2), 1640-1644.

[15] Lu G, Duan Y Y, Wang X D, Lee D J. Internal flow in evaporating droplet on heated solid surface. International Journal of Heat and Mass Transfer, 2011, 54(19-20), 4437-4447.

[16] Mandal D K, Bakshi S. Internal circulation in a single droplet evaporating in a closed chamber. International Journal of Multiphase Flow, 2012, 42, 42-51.

[17] Johnson A I, Hamielec A E, Houghton W T. An experimental study of mass transfer with chemical reaction from single gas bubbles. Can. J. Chem. Eng., 1967, 45(3), 140-144.

[18] Watada H A, Hamielec A E, Johnson A I. A theoretical study of mass transfer with chemical reactions in drops. The Canadian Journal of Chemical Engineering, 1970, 48(3), 255-261.

[19] Chen W H. Unsteady absorption of sulfur dioxide by an atmospheric water droplet with internal circulation. Atmospheric Environment, 2001, 35: 2375-2393.

[20] Chen W H. Dynamics of sulfur dioxide absorption in a raindrop falling at terminal velocity. Atmospheric Environment, 2001, 35(28), 4777-4790.

[21] Chen W H, Chen Y Y, Hung C I. A simplified model of predicting $\mathrm{SO}_{2}$ absorption by single atmospheric raindrops with chemical dissociation and internal circulation. Aerosol and Air Quality Research, 2011, 11, 860-872. 
[22] Chen W H, Tsai M H, Hung C I. Numerical prediction of $\mathrm{CO} 2$ capture process by a single droplet in alkaline spray. Applied Energy, 2013, 109, 125-134.

[23] Elperin T, Fominykh A. Conjugate mass transfer during gas absorption by falling liquid droplet with internal circulation. Atmospheric Environment, 2005, 39(25), 4575-4582.

[24] Elperin T, Fominykh A, Krasovitov B. Scavenging of soluble gases by evaporating and growing cloud droplets in the presence of aqueous-phase dissociation reaction. Atmospheric Environment, 2010, 42(13), 3076-3086.

[25] Hsiang L P, Faeth G M. Near-Limit and secondary breakup. International Journal of Multiphase Flow, 1992, 18(5): 635-652.

[26] Sundararajan T, Ayyaswamy P S. Hydrodynamics and heat transfer associated with condensation on a moving drop: solutions for intermediate Reynolds numbers. Journal of Fluid Mechanics, 1984, 149: 33-58.

[27] Patankar S R. Numerical Heat Transfer and Fluid Flow. Hemisphere, Washington, DC.

[28] Grove A S, Shair F H, Petersen E E, Acrivos A. An experimental investigation of the steady separated flow past a circular cylinder. Journal of Fluid Mechanics, 1964, 19(1), 60-85.

[29] Prakash S, Sirignano W A. Liquid fuel droplet heating with internal circulation. International Journal of Heat and Mass Transfer, 1978, 21(7): 885-895.

[30] Wong S C, Lin A C. Internal temperature distributions of droplets vaporizing in high-temperature convective flows. Journal of Fluid Mechanics, 1992, 237: 671-678. 